\title{
Permeable Surface Corrections for Ffowcs Williams and Hawkings Integrals
}

\author{
David P. Lockard* and Jay H. Casper* \\ NASA Langley Research Center, Hampton, VA 23681
}

\begin{abstract}
The acoustic prediction methodology discussed herein applies an acoustic analogy to calculate the sound generated by sources in an aerodynamic simulation. Sound is propagated from the computed flow field by integrating the Ffowcs Williams and Hawkings equation on a suitable control surface. Previous research suggests that, for some applications, the integration surface must be placed away from the solid surface to incorporate source contributions from within the flow volume. As such, the fluid mechanisms in the input flow field that contribute to the far-field noise are accounted for by their mathematical projection as a distribution of source terms on a permeable surface. The passage of nonacoustic disturbances through such an integration surface can result in significant error in an acoustic calculation. A correction for the error is derived in the frequency domain using a frozen gust assumption. The correction is found to work reasonably well in several test cases where the error is a small fraction of the actual radiated noise. However, satisfactory agreement has not been obtained between noise predictions using the solution from a three-dimensional, detached-eddy simulation of flow over a cylinder.
\end{abstract}

\section{Nomenclature}

$\begin{array}{ll}c & \text { speed of sound } \\ f & \text { integration surface defined by } f=0 \\ F_{i} & \text { dipole source terms } \\ H & \text { Heaviside function } \\ i & \text { imaginary unit, } \sqrt{-1} \\ k & \text { wavenumber, } \omega / c_{o} \\ M_{i} & \text { local source Mach number vector, } v_{i} / c \\ M & \text { Mach number, }\left|M_{i}\right| \\ \hat{n}_{i} & \text { outward directed unit normal vector } \\ p & \text { pressure } \\ \mathbf{Q} & \text { monopole source term } \\ t & \text { time } \\ u_{i} & \text { Cartesian fluid velocity components } \\ v_{i} & \text { Cartesian surface velocity components } \\ x, y, z & \text { Cartesian observer coordinates }\end{array}$

$$
\begin{array}{ll}
\text { Greek: } & \\
\beta & \sqrt{1-M^{2}} \\
\delta(f) & \text { Dirac delta function } \\
\delta_{i j} & \text { Kronecker delta } \\
\rho & \text { fluid density } \\
\xi, \eta, \zeta & \text { Cartesian source coordinates }
\end{array}
$$

\section{Superscript:}

' perturbation quantity (e.g. $\rho^{\prime}=\rho-\rho_{o}$ )

Subscript:

o freestream quantity

\section{Introduction}

The physics-based, airframe noise prediction methodology under investigation is a hybrid of aeroacoustic theory and computational fluid dynamics (CFD). The near-field aerodynamics associated with an airframe component are simulated to obtain the source input to an acoustic analogy that propagates sound to the far field. The acoustic analogy employed within this current framework is that of Ffowcs Williams and Hawkings, ${ }^{1}$ who extended the analogies of Lighthill ${ }^{2}$ and Curle $^{3}$ to the formulation of aerodynamic sound generated by a surface in arbitrary motion. This hybrid approach has been successfully demonstrated in the prediction of aerodynamic noise from various airframe components in landing configuration, such as high-lift wing devices ${ }^{4-6}$ and undercarriages. ${ }^{7-9}$

The Ffowcs Williams and Hawkings (FW-H) equation formulates aerodynamic noise in terms of sources that are distributed on a control surface and throughout the volume external to that surface. Because airframe noise is typically predicted for flows at low Mach number $(\approx 0.2)$, it may appear reasonable to assume that the only significant acoustic

*Aerospace Technologist, Computational AeroSciences Branch, Mail Stop 128, Senior Member, AIAA 
sources are those associated with the solid surface of the aircraft. However, previous research suggests that there are airframe noise problems in which flow-volume sources cannot be neglected. For example, Singer et al. ${ }^{4}$ published slat noise results they obtained by integrating the FW-H equation on various surfaces within a two-dimensional (2-D) Navier-Stokes simulation for a high-lift wing profile. Their results ${ }^{4}$ clearly suggested that the flow in the slat-cove region was contributing significantly to the far field. Moreover, this suggestion by Singer $e t$ al. ${ }^{4}$ has been supported by more recent 2-D slat noise predictions ${ }^{6}$ in which the flow-volume source distribution was directly evaluated by the volume "quadrupole") integral in the FW-H equation.

Note that the 2-D volume-source integration in reference 6 was an exercise to demonstrate the potential importance of the slat-cove flow in future predictions and design guidance. Clearly, the calculation of such volume integrals on a routine basis for three-dimensional (3-D) problems is impractical. Therefore, if a given problem contains nonnegligible flow-volume sources, the FW-H equation should be integrated on a permeable surface that is sufficiently far from the solid body to incorporate the acoustic contribution of those sources, thereby eliminating the need to directly evaluate the FW-H volume integral. At first glance, this approach presents a clear strategy to implement a robust and economical FW-H integration algorithm within the current prediction methodology. However, the movement of fluid disturbances through an integration surface can result in erroneous, nonphysical noise that can seriously impair an acoustic prediction. A simple example of this boundary error phenomenon was demonstrated in reference 6, even though the flow structure in that problem is known to be nonacoustic.

For the 2-D slat noise predictions in reference 6, this boundary-error issue was avoided by a judicious choice for the location of the FW-H integration surface. However, the flow fields associated with trailing edge flaps and undercarriages can generate strong vortical wakes that will eventually exit the downstream boundary of any practical control volume. These wakes are not a problem if the radiated noise associated with these components (or future components) is due almost entirely to source distributions on the solid surface. In such a case, the noise is reliably predicted by integrating the FW-H equation on the solid surface only. However, consider the situation where separate FW-H calculations are performed with a solid surface and a permeable surface with the following conditions:

i. The two acoustic predictions are significantly different in amplitude and/or directivity.

ii. The permeable integration surface, or some part of it, is located in a region of nonuniform flow.

The difference referred to in condition i could be the result of a flow-volume source that is properly included in the permeable surface integration, or the boundary error that could be associated with condition ii. Under these conditions, there is no way to be sure of the distributive nature of the acoustic sources in the flow simulation, without resorting to a expensive FW-H volume integral calculation, which would require access to the volume data which is rarely available. Furthermore, Condition (ii) is difficult to avoid, in general. Although the computational domain of a flow calculation would ideally extend to a surface where the flow field is uniform, this is rarely the case, in practice. A detailed discussion on the placement of the integration surface can be found in reference 10.

The goal of the present research is to develop boundary correction techniques for the permeable surface integral of FW-H equation that will diminish, if not eliminate, the error that results from Condition (ii). The FW-H equation and its integral solutions are presented in a manner that applies to both solid and permeable surfaces.

Other researchers have attempted to address the error associated with the passing of Lighthill stresses through the boundary of a truncated control volume. Some specific boundary-correction techniques have been proposed by previous authors, ${ }^{11,12}$ but many approaches are only applicable to truncating a volume quadrupole integration because they rely on damping out the hydrodynamic disturbances before the end of the domain. For a permeable-surface FW-H calculation, the acoustic signal would also be lost. Wang et al. ${ }^{13}$ developed a correction based on a frozen convection assumption that resulted in a boundary flux correction for Curle's equation. In the current work, we employ the frozen gust assumption and develop corrections for the frequency domain form of the FW-H equation. Several test cases are used to demonstrate the efficacy and need for the approach.

\section{Acoustic Equations}

The FW-H equation can be written in differential form ${ }^{14}$ as

$$
\left(\frac{\partial^{2}}{\partial t^{2}}-c_{o}^{2} \frac{\partial^{2}}{\partial x_{i} \partial x_{i}}\right)\left(H(f) \rho^{\prime}\right)=\frac{\partial^{2}}{\partial x_{i} \partial x_{j}}\left(T_{i j} H(f)\right)-\frac{\partial}{\partial x_{i}}\left(F_{i} \delta(f)\right)+\frac{\partial}{\partial t}(Q \delta(f))
$$

where

$$
T_{i j}=\rho u_{i} u_{j}+P_{i j}-c_{o}^{2} \rho^{\prime} \delta_{i j}, \quad F_{i}=\left(P_{i j}+\rho u_{i}\left(u_{j}-v_{j}\right)\right) \frac{\partial f}{\partial x_{j}}, \text { and }
$$




$$
Q=\left(\rho_{o} v_{i}+\rho\left(u_{i}-v_{i}\right)\right) \frac{\partial f}{\partial x_{i}}
$$

The dipole term $F_{i}$ involves an unsteady force, and $Q$ gives rise to a monopole-type contribution that can be thought of as an unsteady mass addition. The function $f=0$ defines the surface outside of which the solution is desired. The normalization $|\nabla f|=1$ is used for $f$. The total density and pressure are given by $\rho$ and $p$, respectively. The fluid velocities are $u_{i}$, while the $v_{i}$ represent the velocities of the surface $f$. The Kronecker delta, $\delta_{i j}$, is unity for $i=j$ and zero otherwise. The ambient speed of sound is denoted by $c_{o}$. A prime is used to denote a perturbation quantity relative to the free stream conditions denoted by the subscript $o$. The Cartesian coordinates and time are $x_{i}$ and $t$, respectively. The usual convention, which is followed here, involves a quiescent ambient state with $f$ prescribed as a function of time so that it always surrounds a moving source region of interest. $H(f)$ is the Heaviside function which is unity for $f>0$ and zero for $f<0$. The derivative of the Heaviside function $H^{\prime}(f)=\delta(f)$ is the Dirac delta function, which is zero for $f \neq 0$, but yields a finite value when integrated over a region including $f=0$. The inviscid part, $P_{i j}=p \delta_{i j}$, of the compressive stress tensor $P_{i j}$ is used in this work.

The FW-H equation is an exact rearrangement of the Navier-Stokes equations, and allows one to determine the acoustic signal at distant observer locations if the details of the source region are already known. Hence, the NavierStokes equations still need to be solved, but only where nonlinear and viscous effects are important. All of the linear propagation can be determined by the FW-H equation. For 3-D flows, the time-domain FW-H formulations developed by Farassat ${ }^{15}$ are efficient and amenable to numerical computations. Some additional efficiency can be obtained by restricting the source to uniform, rectilinear motion. Furthermore, the equation can be solved in the frequency domain, which can be useful if one is only interested in analyzing certain frequencies. Under the assumption $f=f(\mathbf{x}+\mathbf{U} t)$, where the components of $\mathbf{U}$ are constant velocities describing the motion of the surface, the frequency domain solution of the FW-H equation can be written in the form ${ }^{16,17}$

$$
H(f) c_{o}^{2} \rho^{\prime}(\mathbf{y}, \omega)=I_{T}+I_{L}+I_{Q}
$$

where

$$
I_{T}=-\int_{f=0} i \omega Q_{n}(\boldsymbol{\xi}, \omega) G(\mathbf{y} ; \boldsymbol{\xi}) d s \text { and } I_{L}=-\int_{f=0} F_{i}(\boldsymbol{\xi}, \omega) \frac{\partial G(\mathbf{y} ; \boldsymbol{\xi})}{\partial y_{i}} d s
$$

For a moving surface, the $u_{i}$ are perturbation quantities and

$$
F_{i}=\left(p \delta_{i j}+\rho\left(u_{i}-U_{i}\right)\left(u_{j}+U_{j}\right)+\rho_{o} U_{i} U_{j}\right) \hat{n}_{j}, \quad Q_{n}=\left(\rho\left(u_{i}+U_{i}\right)-\rho_{o} U_{i}\right) \hat{n}_{i} .
$$

The volumetric quadrupole term is denoted by $I_{Q}$ and includes effects such as nonlinear propagation and refraction and can be expressed as

$$
I_{Q}=-\int_{f>0} T_{i j}(\boldsymbol{\xi}, \omega) \frac{\partial^{2} G(\mathbf{y} ; \boldsymbol{\xi})}{\partial \xi_{i} \partial \xi_{j}} d \boldsymbol{\xi}
$$

Equation 3 is valid in two or three dimensions depending on the form of the free-field Green's function. Denoting the source coordinates as $\xi_{i}$ and the observer position as $y_{i}$, the 2-D Green's function for flow in the $y_{1}$ direction is ${ }^{16}$

$$
G(\mathbf{y} ; \boldsymbol{\xi})=\frac{i}{4 \beta} \exp ^{\left(i M k\left(y_{1}-\xi_{1}\right) / \beta^{2}\right)} H_{o}^{(2)}\left(\frac{k}{\beta^{2}} \sqrt{\left(y_{1}-\xi_{1}\right)^{2}+\beta^{2}\left(y_{2}-\xi_{2}\right)^{2}}\right)
$$

The Mach number is denoted as $M=\left|U_{i}\right| / c_{o}=U_{1} / c_{o}$. The 3-D Green's function ${ }^{18}$ for flow in the $y_{1}$ direction is

$$
G(\mathbf{y} ; \boldsymbol{\xi})=\frac{-1}{4 \pi d} \exp ^{\left(-i k\left(d-M\left(y_{1}-\xi_{1}\right)\right) / \beta^{2}\right)}
$$

where $d=\sqrt{\left(y_{1}-\xi_{1}\right)^{2}+\beta^{2}\left(y_{2}-\xi_{2}\right)^{2}+\beta^{2}\left(y_{3}-\xi_{3}\right)^{2}}$ and $k=\omega / c_{o}$.

In many applications, the volumetric contribution is small and is neglected. However, hydrodynamic disturbances produce a contribution to the thickness $\left(I_{T}\right)$ and loading $\left(I_{L}\right)$ terms when they pass through porous surfaces. These contributions are canceled by the volume quadrupole, but the calculation of this time-dependent integral is impractical on a routine basis. In many circumstances, the errors are small and can be ignored. However, there are classes of problems where the error is commensurate with the true acoustic signal. Nonetheless, it is desirable to avoid the volume quadrupole calculation because of the expense. 


\section{Quadrupole Correction}

In many flows, hydrodynamic disturbances simply convect with the mean flow. Under the assumption of frozen convection, each of the primitive variables, as well as the Lighthill stress tensor, are governed by a simple convection equation. For simplicity, the mean flow is assumed to be in the $y_{1}$ direction, which leads to

$$
\frac{\partial T_{i j}}{\partial t}+M \frac{\partial T_{i j}}{\partial y_{1}}=0
$$

An application of a Fourier transform produces the relation

$$
T_{i j}=\frac{i M}{\omega} \frac{\partial T_{i j}}{\partial y_{1}}
$$

The relation in equation 10 can be used to replace $T_{i j}$ in equation 6 to obtain

$$
I_{Q}=-\int_{f>0} \frac{i M}{\omega} \frac{\partial T_{i j}}{\partial \xi_{1}} \frac{\partial^{2} G(\mathbf{y} ; \boldsymbol{\xi})}{\partial \xi_{i} \partial \xi_{j}} d \boldsymbol{\xi}
$$

Integrating by parts yields

$$
I_{Q}=-\int_{f=0} \frac{i M}{\omega} T_{i j} \frac{\partial^{2} G(\mathbf{y} ; \boldsymbol{\xi})}{\partial \xi_{i} \partial \xi_{j}} d s+\int_{f>0} \frac{i M}{\omega} T_{i j} \frac{\partial}{\partial \xi_{1}}\left(\frac{\partial^{2} G(\mathbf{y} ; \boldsymbol{\xi})}{\partial \xi_{i} \partial \xi_{j}}\right) d \boldsymbol{\xi}
$$

Repeated integration by parts produces the series

$$
I_{Q}=-\left(\frac{-i M}{\omega}\right)^{N} \int_{f>0} T_{i j} \frac{\partial^{N}}{\partial \xi_{1}^{N}}\left(\frac{\partial^{2} G(\mathbf{y} ; \boldsymbol{\xi})}{\partial \xi_{i} \partial \xi_{j}}\right) d \boldsymbol{\xi}+\sum_{n=1}^{N}\left(\frac{-i M}{\omega}\right)^{n} \int_{f=0} T_{i j} \frac{\partial^{(n-1)}}{\partial \xi_{1}^{(n-1)}}\left(\frac{\partial^{2} G(\mathbf{y} ; \boldsymbol{\xi})}{\partial \xi_{i} \partial \xi_{j}}\right) d s
$$

The first term in the series is related to the quadrupole boundary correction developed by Wang et al. ${ }^{13}$ Clearly, the series has a strong dependence on the Mach number of the flow, and it also appears to be dependent on the frequency $\omega$. However, the Green's function also depends on $M$ and $\omega$, so these parameters also appear in the derivatives of $G$. Based on several test cases, the influence of $M$ seems much more important to the convergence of the series. A notable exception is in the near-field where series convergence for all Mach numbers is often poor for very low frequencies.

Although the series only involves an integral over the projection of the boundary surface onto a plane normal to the flow direction, repeated derivatives of the Green's function are required. The second derivatives of $G$ in two dimensions have been report by Gloerfelt et al. ${ }^{19}$ A symbolic algebra package can be used to obtain the higher derivatives. The higher derivatives are quite complicated and nontrivial to calculate. Nonetheless, computing the quadrupole boundary correction for a few terms is still considerably less expensive than computing the volume quadrupole.

Experimentation has demonstrated that using the local, time-averaged value of the flow velocity, wherever $M$ appears explicitly in equation 13, is beneficial. The improvement has been found to be most important in 3-D problems.

\section{2-D Convecting Vortex}

A convecting vortex ${ }^{20,21}$ is used to assess the effectiveness of the quadrupole boundary correction. An exact solution to the compressible Euler equation is

$$
\begin{aligned}
p & =\frac{1}{\gamma}\left(1-a_{2} \exp \left(1-r^{2}\right)\right)^{\frac{\gamma}{\gamma-1}}, \quad \rho={\frac{p}{P_{o}}}^{\frac{1}{\gamma}} \\
u & =M-a_{0} a_{1} y \exp \left(\left(1-r^{2}\right) / 2\right) \\
v & =a_{0} a_{1}(x-M t) * \exp \left(\left(1-r^{2}\right) / 2\right)
\end{aligned}
$$

where $r^{2}=(x-M t)^{2}+y^{2}, a_{0}=1, a_{1}=1 /(2 \pi)$, and $a_{2}=1 / 2(\gamma-1) a_{1}^{2} a_{0}^{2}$. Here, $x$ and $y$ are the 2-D Cartesian coordinates. The pressure contours of the vortex are shown in figure 1(a). The porous FW-H surface used in the 2-D code FWH2D ${ }^{16}$ is shown in red. The FWH2D codes solves equation 3, assuming $I_{Q}=0$. The surface is a square, with each side of length 10 units. All the results in the paper are dimensionless with some reference length, $L$ the speed of 


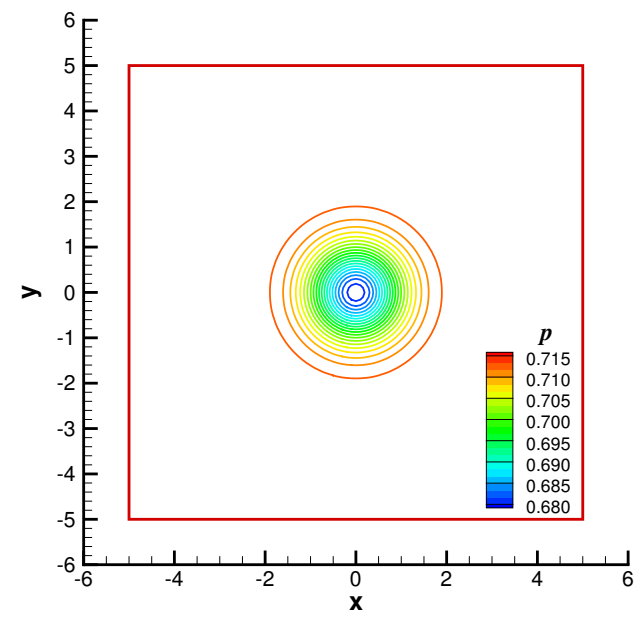

(a) Pressure of vortex

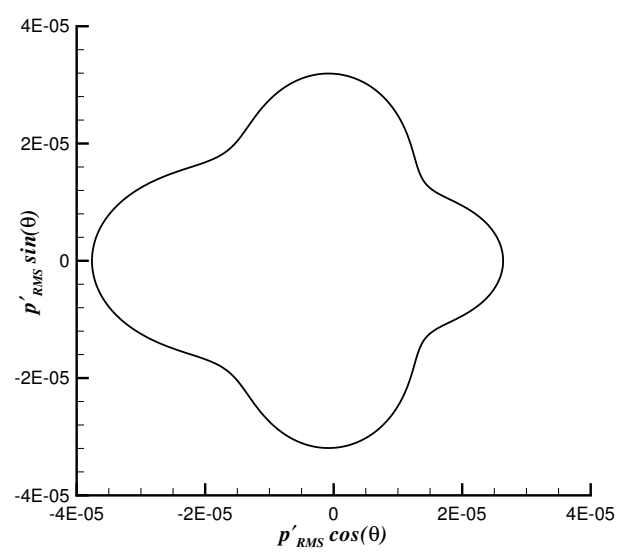

(b) Directivity

Figure 1. (a) Pressure contours of the vortex at $t=0$. The FW-H surface is in red. (b) Directivity of the erroneous noise from an FW-H 2-D calculation $\left(I_{T}+I_{L}\right)$ when a compressible vortex passes through a porous surface. Observers are at a radius of 100, and $M=0.2$.

sound, $c_{o}$ and the density, $\rho$ used as reference quantities. The directivity pattern calculated by FWH2D for the vortex convecting through the surface at $M=0.2$ is presented in figure 1(b). The exact solution is zero for all time, so the computed noise is all error. Whether such errors are important would depend on the magnitude of the true acoustic signal in the problem.

In figure $2 \mathrm{a}$ and $\mathrm{b}$, the FWH2D solution at $M=0.2$ is compared with the approximations to $I_{Q}$ from equation 13 using one, two, and three terms in the series expansion. At $M=0.2$, two terms are needed to correctly cancel the erroneous part of the signal, and there is little difference in the quality of the correction in the upstream and downstream directions. At $M=0.3$, figure 3(a) shows that the downstream correction monotonically approaches the FWH2D value; however, the upstream correction oscillates around the FWH2D solution as seen in figure 3(b). Nonetheless, the correction is quite good with three terms. Figure $4 \mathrm{a}$ and $\mathrm{b}$ shows similar results for $M=0.4$, although the convergence in the upstream direction is very slow, and even three terms are insufficient. Although the results for the downstream observer at $M=0.6$ in figure 5(a) are still quite good, figure 5(b) shows that the series is actually divergent for the upstream observer. The divergence occurs smoothly as the observer is moved from the downstream to upstream location. The reason for the difference between the upstream and downstream directions is unclear, but use of the correction should clearly be restricted to low Mach numbers.

\section{2-D Cylinder Shedding}

The flow around a circular cylinder is used to investigate the effectiveness of the quadrupole boundary correction for problems with a real acoustic signal. The input data for the acoustic calculation is obtained from a time-dependent CFD solution using the code CFL3D. ${ }^{22,23}$ CFL3D was developed at NASA Langley Research Center to solve the 3-D, time-dependent, thin layer Reynolds-averaged Navier-Stokes (RANS) equations using a finite-volume formulation. The code was operated in a 2-D mode for the current calculations. The unsteady RANS calculation employed the shear stress transport (SST) $k-\omega$ turbulence model of Menter, ${ }^{24,25}$ which was developed for steady flow. The multiblock grid contains 140,000 grid points. The Mach number of the flow is 0.15 , and the Reynolds number based on the cylinder diameter is 1.2 million. The code was run until a periodic steady-state was obtained before sampling the solution. The time step was decreased until no significant change was observed. The dimensionless time step is 0.05 , giving nearly 450 time steps/period of the primary shedding frequency. Twenty-five subiterations are used for every time step to promote convergence of the dual-time algorithm. The solution is sampled at every other time step until 4096 samples are acquired.

The instantaneous pressure contours around the cylinder are shown in figure 6 where the vortex street behind the cylinder is apparent. The surfaces used to sample data for the FWH2D code are superimposed on the solution. The cylinder surface is denoted as S, and the two porous surfaces as P1 and P2.

The directivities associated with the FWH2D solutions using the three surfaces are shown in figure 7(a). The ob- 


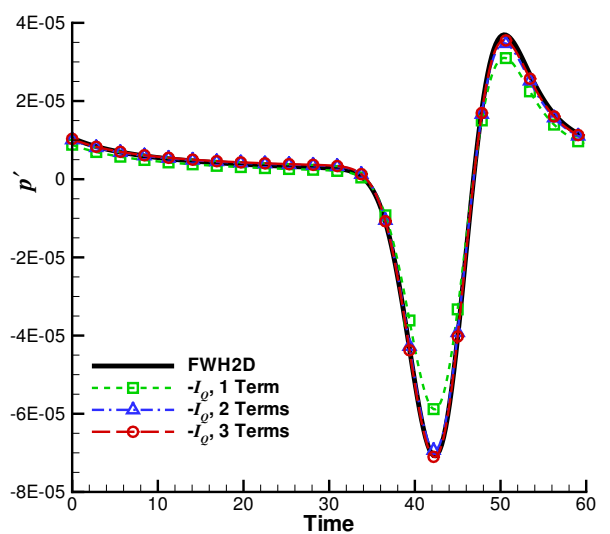

(a) Downstream Observer

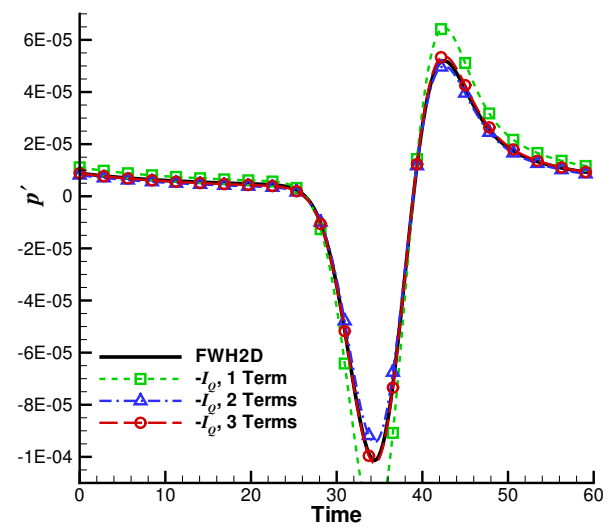

(b) Upstream Observer

Figure 2. Comparison of the quadrupole correction $\left(I_{Q}\right)$ to the erroneous noise from an FWH2D calculation $\left(I_{T}+I_{L}\right)$ when a compressible vortex passes through a porous surface. Observers are at a radius of 100, and $M=0.2$.

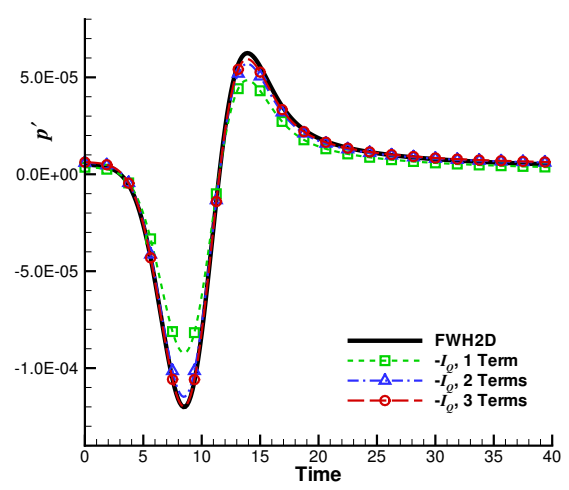

(a) Downstream Observer

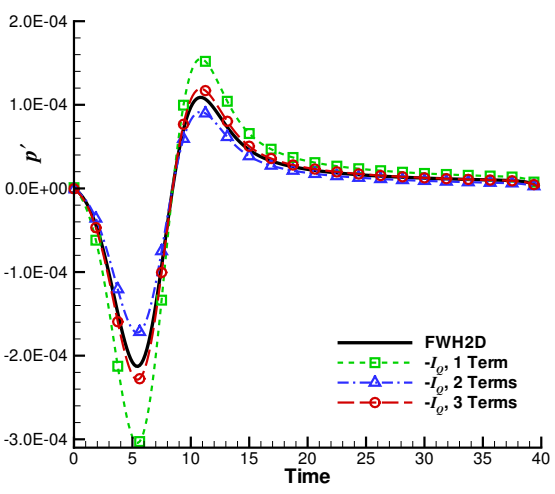

(b) Upstream Observer

Figure 3. Comparison of the quadrupole correction $\left(I_{Q}\right)$ to the erroneous noise from an FWH2D calculation $\left(I_{T}+I_{L}\right)$ when a compressible vortex passes through a porous surface. Observers are at a radius of 100, and $M=0.3$.

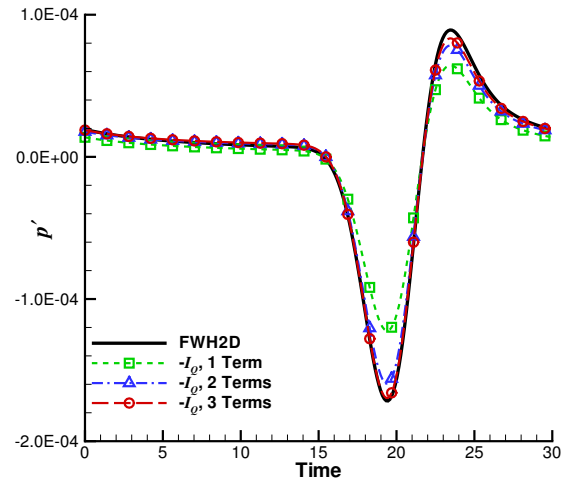

(a) Downstream Observer

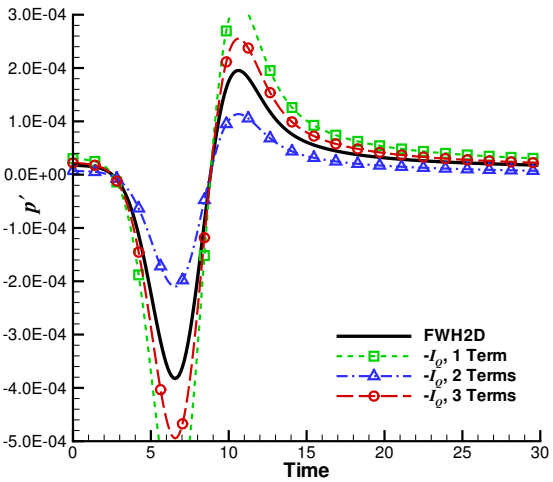

(b) Upstream Observer

Figure 4. Comparison of the quadrupole correction $\left(I_{Q}\right)$ to the erroneous noise from an FWH2D calculation $\left(I_{T}+I_{L}\right)$ when a compressible vortex passes through a porous surface. Observers are at a radius of $\mathbf{1 0 0}$, and $M=0.4$. 


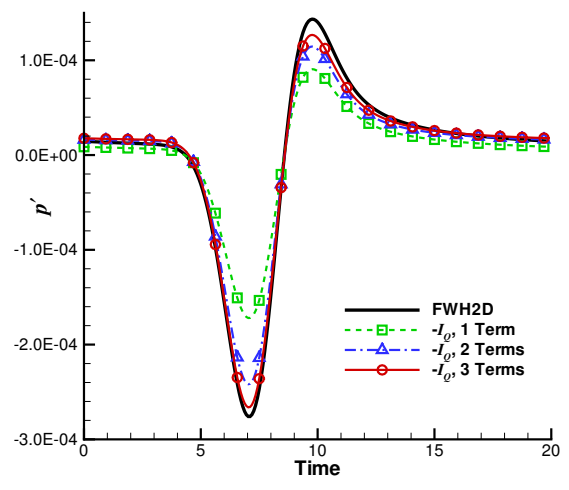

(a) Downstream Observer

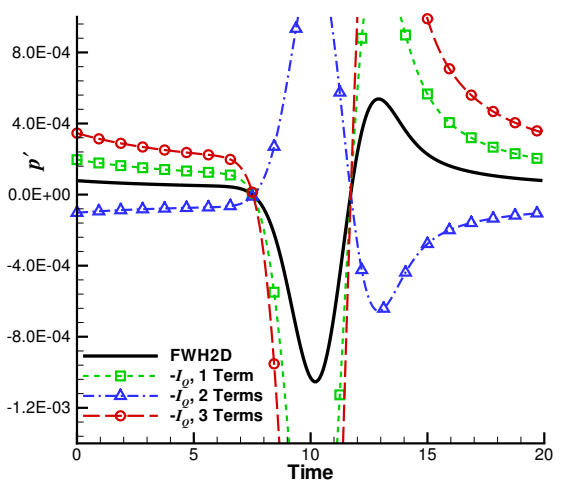

(b) Upstream Observer

Figure 5. Comparison of the quadrupole correction $\left(I_{Q}\right)$ to the erroneous noise from an FWH2D calculation $\left(I_{T}+I_{L}\right)$ when a compressible vortex passes through a porous surface. Observers are at a radius of 100, and $M=0.6$.

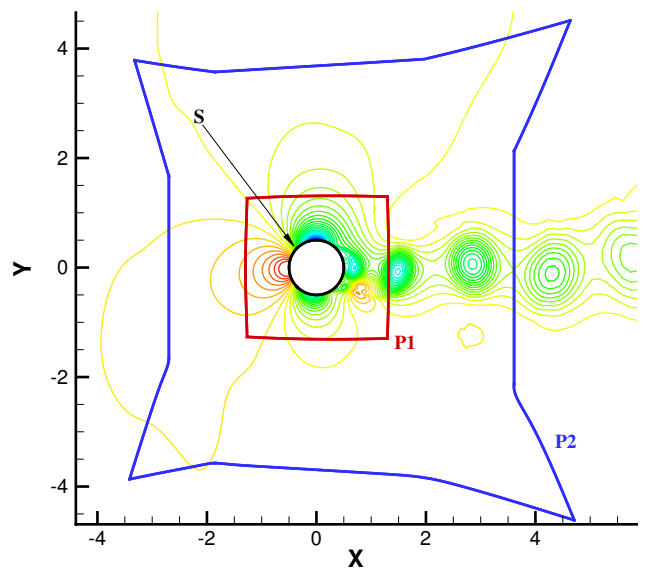

Figure 6. (a) CFL3D computed pressure contours around a circular cylinder in a $M=0.15$ flow. Red and blue lines indicate FW-H surfaces. 
servers are located 100 cylinder diameters from the center of the cylinder. The solutions using the solid surface and inner porous solution actually appear to be in reasonable agreement, but there is some discrepancy with the P2 solution. However, the time histories for an observer at $r=4, \theta=180^{\circ}$ (upstream) in figure 7(b) show that the neither of the porous surface solutions is in good agreement with the solid surface solution. The solid surface solution agrees reasonably well with the directly sampled CFD solution for the upstream observer, but the agreement with the two porous surface solutions is poor. In contrast, reasonable agreement is observed in the the time history comparison in figure 8(b) when the series approximation to $I_{Q}$ is combined with the FWH2D porous surface solution. The CFD and S surface solutions are the same in figures 7(b) 8(b). Most of the discrepancy with the extracted CFD data is caused by some artificial dissipation of the high frequency part of the signal in the CFD calculation. The directivities for the two porous surface solutions are in agreement in figure 8(a), but differ from the solid surface solution. It is possible that there are some real quadrupole effects caused by the acoustic propagation through the nonuniform mean flow around the cylinder. Regardless, the influence of the quadrupole boundary correction is quite small relative to the true noise signal. The authors have investigated several bluff body shedding problems in two dimensions, and the true noise radiation seems to dominate the compute signal, and the erroneous noise is of the same order of magnitude as the real quadrupole effect. However, these effects are problem dependent, and further quantification of these effects for different classes of problems is needed.

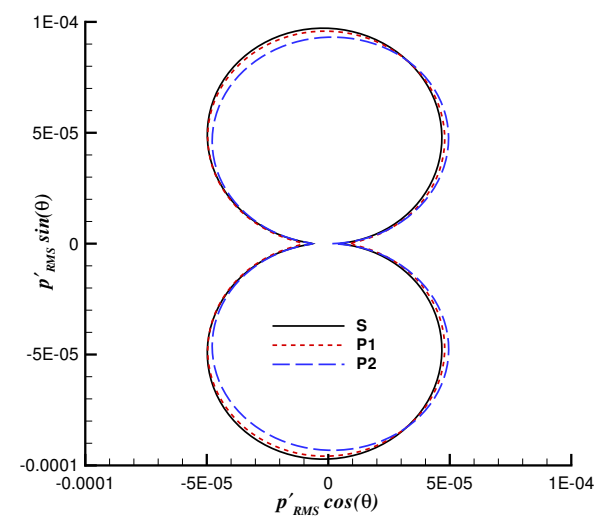

(a) Directivity

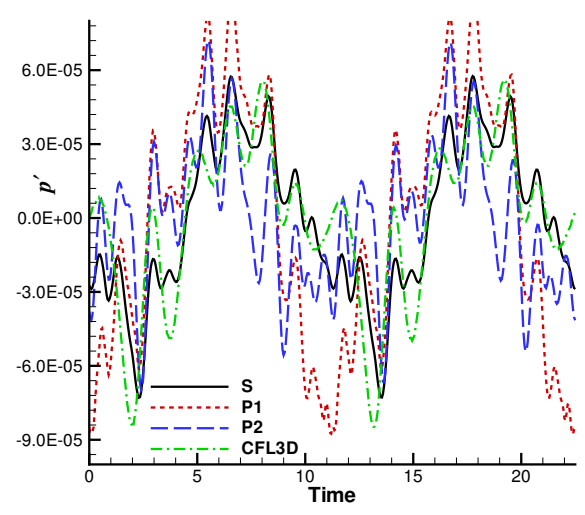

(b) Time History for $r=4, \theta=180^{\circ}$

Figure 7. Comparison of directivities for observers located 100 cylinder diameters from the cylinder and time histories for an upstream observer. FWH2D calculations $\left(I_{T}+I_{L}\right)$ performed with solid surface $\mathbf{S}$ and porous surfaces $\mathbf{P 1}$ and P2. CFD calculation used $M=0.15$.

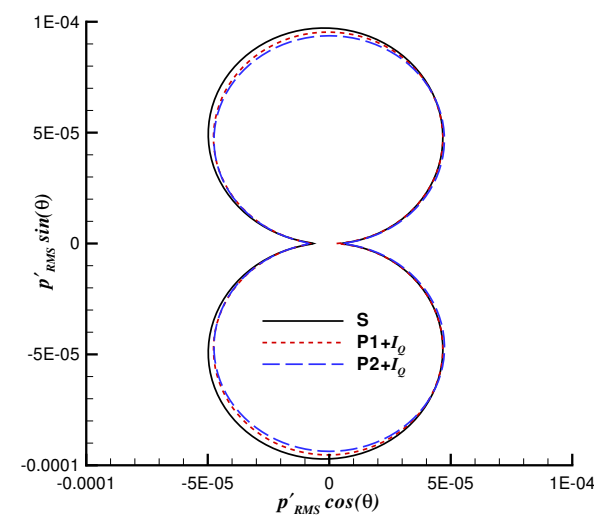

(a) Directivity

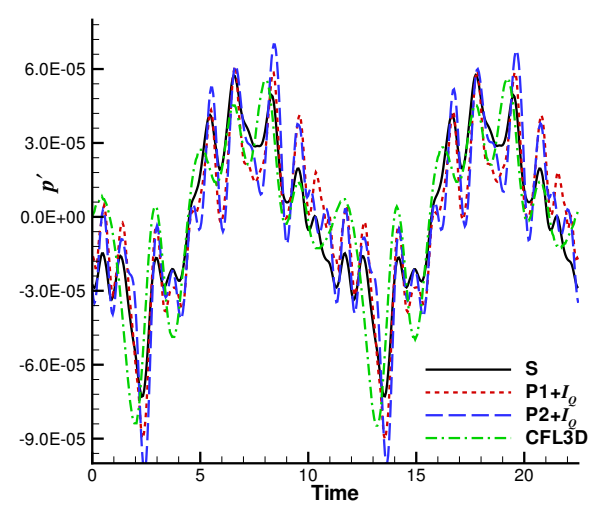

(b) Time History for $r=4, \theta=180^{\circ}$

Figure 8. Quadrupole $\left(I_{Q}\right)$ corrected comparison of directivities for observers located 100 cylinder diameters from the cylinder and time histories for an upstream observer. Calculations performed with solid surface $S$ and porous surfaces P1 and P2. CFD calculation used $M=0.15$. 


\section{3-D Convecting Vortex}

The quadrupole boundary correction has also been implemented in three dimensions. The 2-D, convecting vortex problem $^{20,21}$ was solved in three dimensions by replicating the data in the spanwise direction. The spanwise extent was increased to 10,000 units beyond which negligible variation in the computed solution was observed. Figure 9 shows the time history comparison for $M=0.2$ of the $\mathrm{FWH}_{3} \mathrm{D}^{17}$ solution with the computed quadrupole correction from equation 13. The results are nearly identical to those observed in the 2-D solution in figure 2. Indeed, the 3-D calculations produce the same trends with increasing Mach number. Figure 10 shows that the correction in the upstream direction is divergent just as in the 2-D case. Because the Green's functions in two and three dimensions are completely different, it is likely that the divergence is of a mathematical nature rather than an algebraic or coding error.

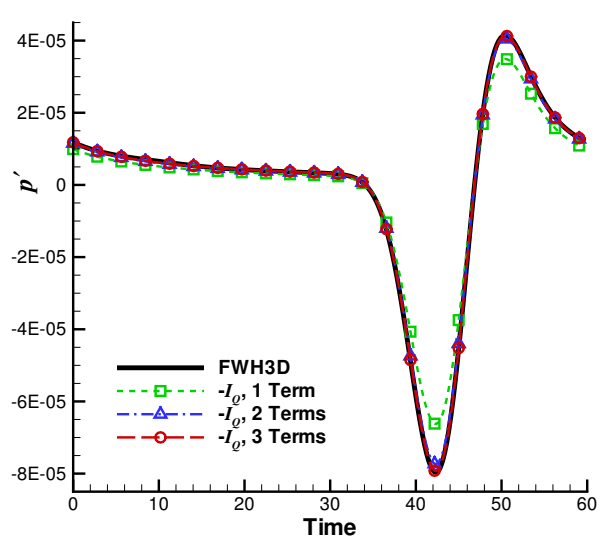

(a) Downstream Observer

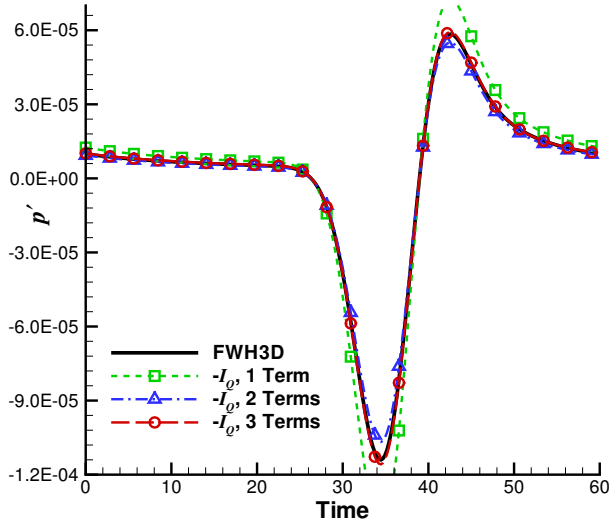

(b) Upstream Observer

Figure 9. Comparison of the quadrupole correction $\left(I_{Q}\right)$ to the erroneous noise from an FWH3D calculation $\left(I_{T}+I_{L}\right)$ when a 2-D, compressible vortex passes through a porous surface. The spanwise extent is 10,000 units. Observers are at a radius of 100, and $M=0.2$.

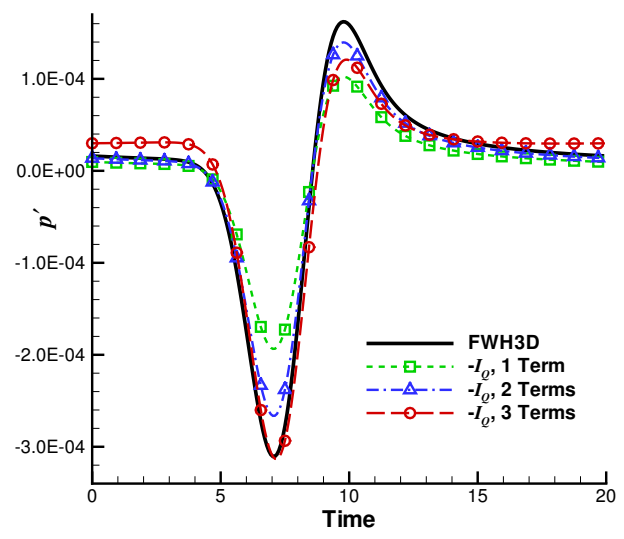

(a) Downstream Observer

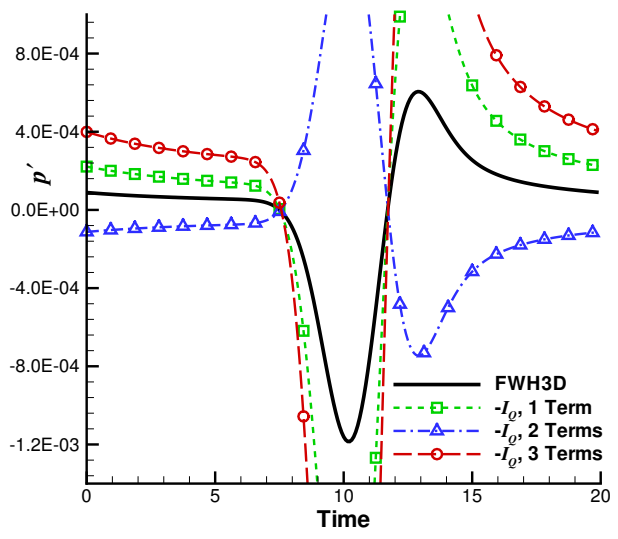

(b) Upstream Observer

Figure 10. Comparison of the quadrupole correction $\left(I_{Q}\right)$ to the erroneous noise from an FWH3D calculation $\left(I_{T}+I_{L}\right)$ when a 2-D, compressible vortex passes through a porous surface. The spanwise extent is $\mathbf{1 0 , 0 0 0}$ units. Observers are at a radius of 100 , and $M=0.6$.

\section{3-D Cylinder Shedding}

To further investigate the utility of the quadrupole boundary correction in three dimensions, CFL3D 22,23 is used to compute the flow around a cylinder with periodicity enforced in the spanwise direction after seven cylinder diameters. The polar grid has 161 points in the radial and azimuthal directions, and 129 points in the spanwise direction. Calcu- 
lations were performed with the Spalart-Allmaras ${ }^{26}$ (S-A) RANS turbulence model and detached-eddy simulation ${ }^{27,28}$ (DES) methodology proposed by Spalart.

The Mach number of the flow is 0.25 , and the Reynolds number based on the cylinder diameter is 100,000 . Although the DES calculation never reaches a completely periodic steady-state, the code was run until time-averaged mean quantities were stationary before sampling the solution. The dimensionless time-step is 0.05 providing over 200 time steps/period of the primary shedding frequency. Twenty-five subiterations are used for every time step to promote convergence of the dual-time algorithm. The solution was sampled at every other time step for a total of 4096 samples. The fluid moves over 100 cylinder diameters during the sampling time.

The instantaneous pressure contours from both the DES and S-A calculations are shown in figure 11. Although there are similarities, the irregularity in the DES solution is much greater, especially in the spanwise direction. The corresponding directivities are shown in figure $12 \mathrm{a}$ and $\mathrm{b}$ for observers at a radius of 100 at midspan of the cylinder. Solid denotes the solution obtained using only the pressure on the surface of the solid cylinder. The porous surface is cylindrical in shape with a radius 1.6. The addition of the quadrupole correction, $I_{Q}$, improves the comparison with the solid surface solution for the S-A case in figure 12(b), but the correction does little to improve the comparison in the DES case shown in 12(a). From animations of the flowfield, it is evident that the variations in the DES solution of the spanwise and cross-stream fluctuations are similar in amplitude and time-scale to those in the convection direction. Hence, the frozen gust assumption may be violated. However, other factors may be contributing to the discrepancy. In figure 13, the directivities for observers at a radius of 7.3 are compared with solutions taken directly from the CFL3D calculations. The CFD solutions are somewhat contaminated by near-field pressure fluctuations associated with wake instabilities and boundary reflections. Only the portion of the directivity that appears sensible are plotted in figure 13 . Although the influence of the turbulence on the solutions is not completely understood, it is clear that the numerics are having a strong influence on the noise predictions. In comparison to the S-A solution, the vortices in the DES case roll-up further downstream producing smaller pressure fluctuations on the cylinder surface. Refining the grid was found to increase the discrepancy.

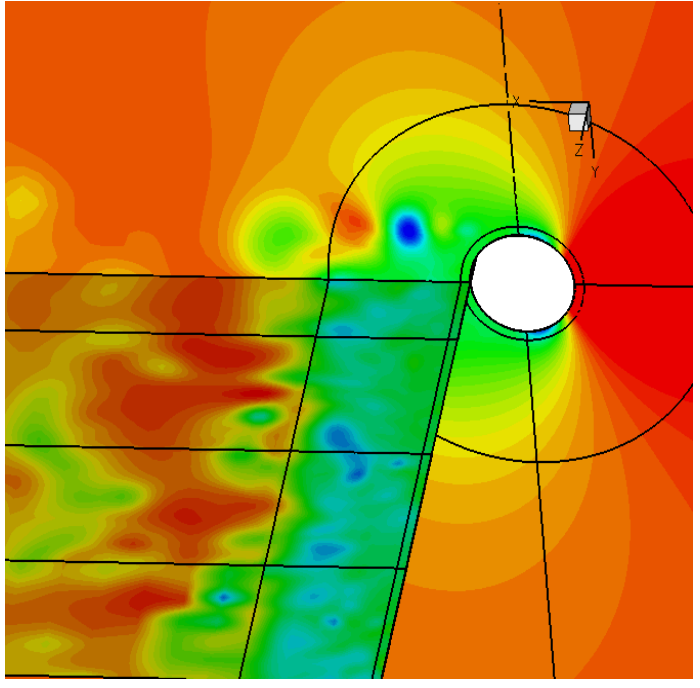

(a) DES

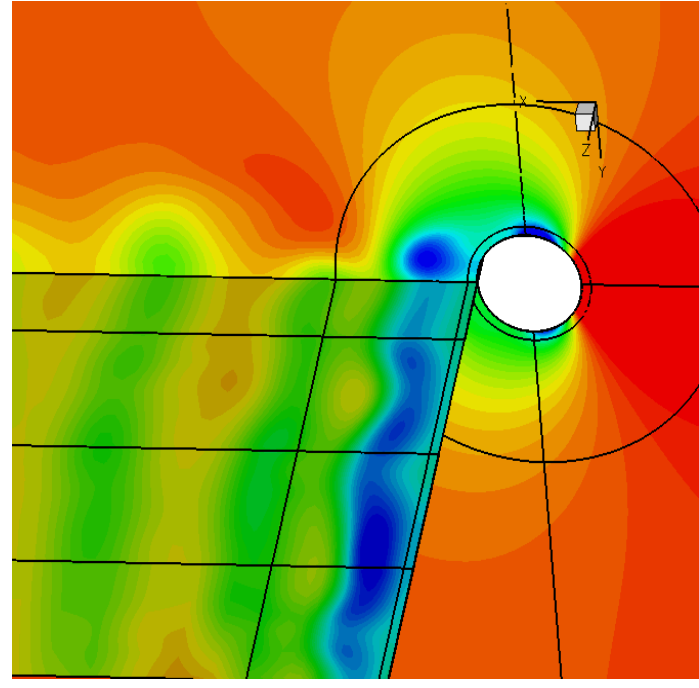

(b) S-A

Figure 11. Comparison of pressure on two planes from CFD calculations of flow over a 3-D cylinder at $M=0.25$. The vertical plane is a normal cut through the cylinder, and the horizontal plane is downstream of the cylinder in the streamwise and spanwise directions. Periodicity was enforced in the span at 7 cylinder diameters. Flow is from right to left.

To further investigate the difference between the predictions using the solid and porous surfaces, the DES case was run further and sampled on two additional porous surfaces. The computed directivities using the additional porous surfaces are shown in figure 14. The surface $\mathrm{P} 2$ has a radius of 1.59 and corresponds to the porous surface used in figures 12 and 13. Surfaces P1 and P3 are at radii of 0.79 and 4.43, respectively. The vortices being shed from the cylinder rollup beyond the innermost surface, P1. Although the location of the porous surface obviously has a strong influence on the calculated results, the overall quality of the simulation is insufficient to determine if the differences are due to real quadrupole sources between the surfaces or numerical error. Both resolution issues and boundary reflections make it difficult to interpret the results.

Comparisons of time histories from the DES case are shown in figure 15. The observers are located 100 cylinder 


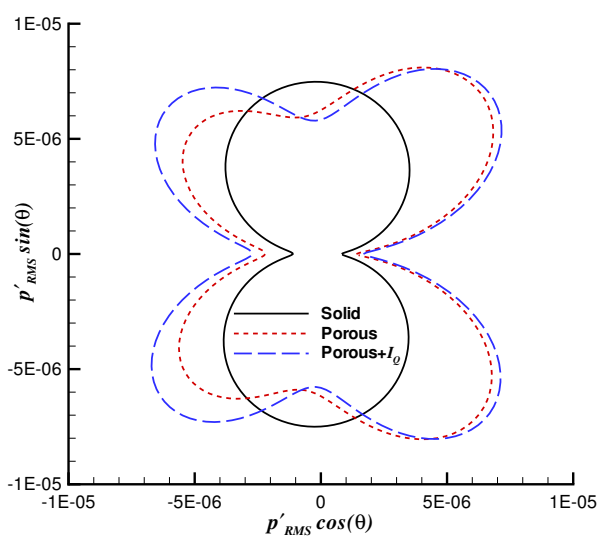

(a) DES

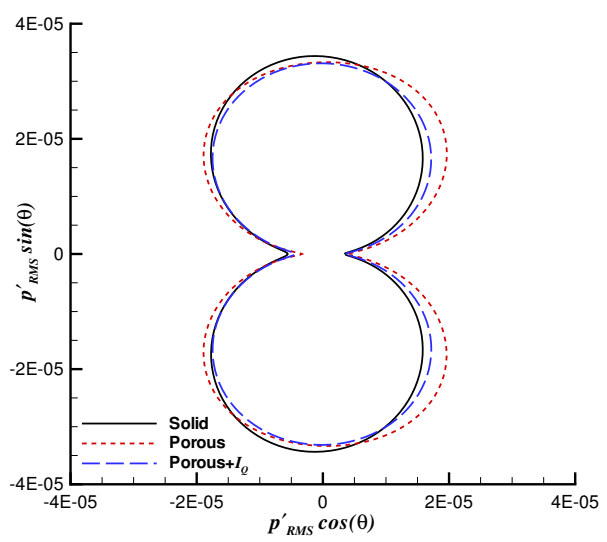

(b) S-A

Figure 12. Comparison of computed directivities from flow over a 3-D cylinder at $M=0.25$. Observers are located at $r=100$.

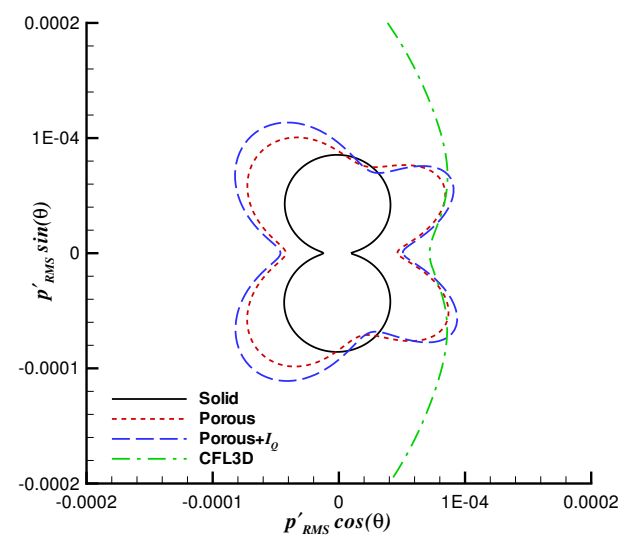

(a) DES

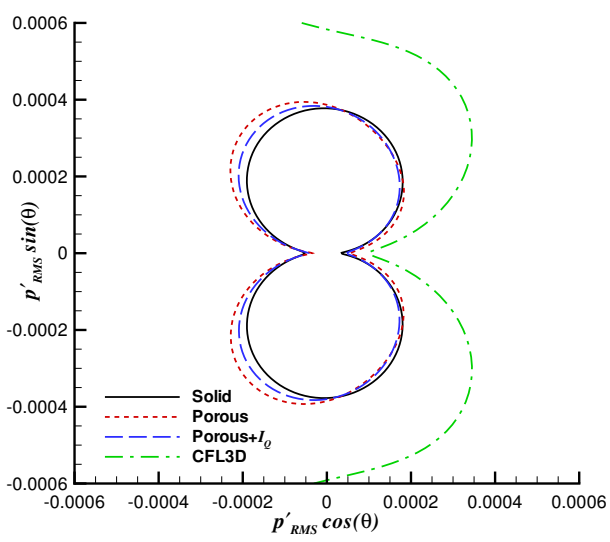

(b) S-A

Figure 13. Comparison of computed directivities from flow over a 3-D cylinder at $M=0.25$. Observers are located at $r=7.3$.

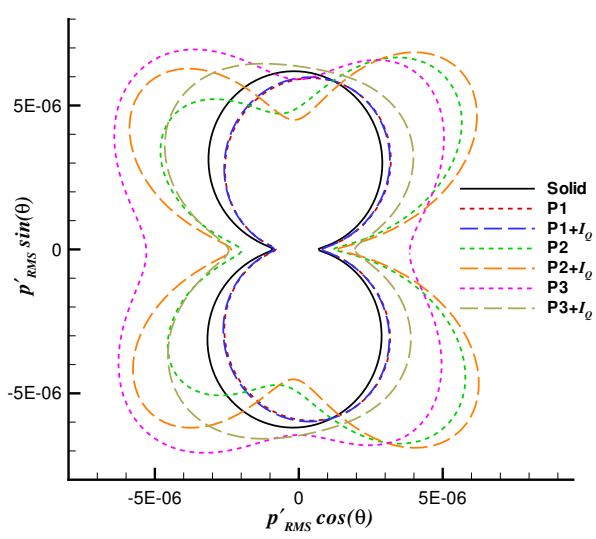

(a) Observers at $r=100$

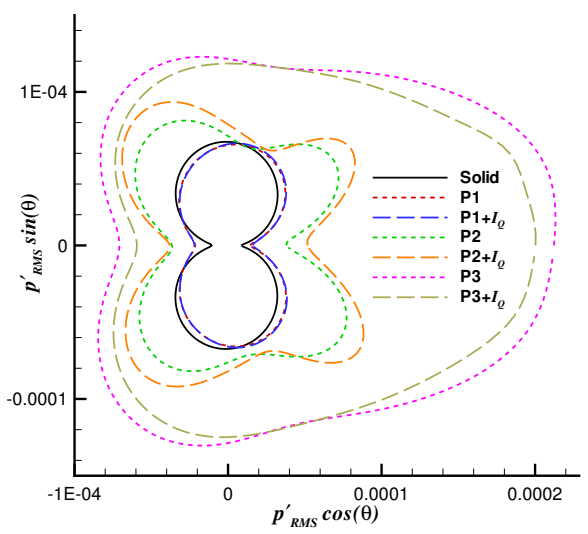

(b) Observers at $r=7.3$

Figure 14. Comparison of computed directivities from DES computations of flow over a 3-D cylinder at $M=0.25$. The radii for the porous surfaces are: $r=0.79$ on P1, $r=1.59$ on P2, and $r=4.43$ on P3. 
diameters from the center of the cylinder, and the angle is measured from the upstream direction. As expected from the directivity plots, the noise predictions using the solid surface data have smaller amplitudes than their porous surface counterparts. The addition of the quadrupole correction has only a slight effect on the solutions.

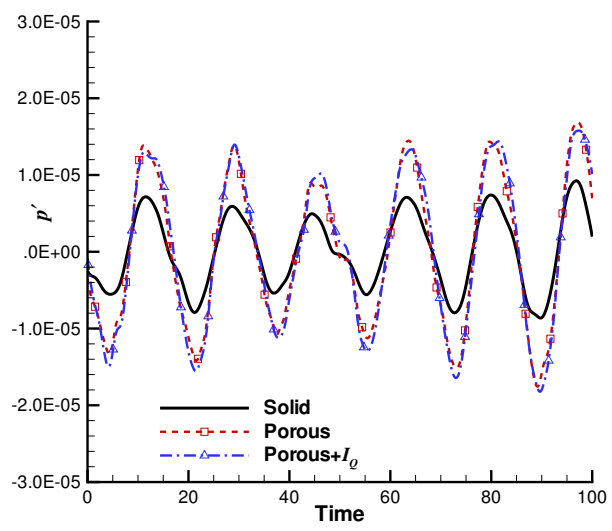

(a) $45^{\circ}$

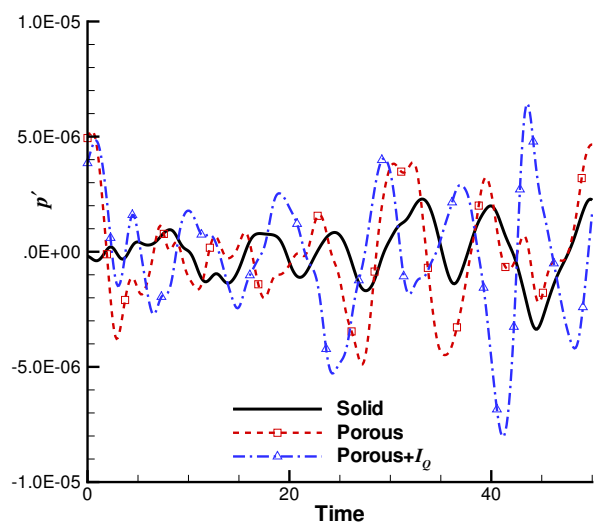

(b) $180^{\circ}$

Figure 15. Time histories of the pressure computed from DES calculations of flow over a 3-D cylinder at $M=0.25$. The observers are located on a radius of 100, and $\theta$ is measured from the upstream direction.

The time histories from the S-A case are shown in figure 16 . At $45^{\circ}$, the correction accounts for nearly all of the discrepancy between the porous and solid surface predictions. At $180^{\circ}$, the solid and porous surface predictions are nearly completely out of phase, but the corrected porous surface solution matches up reasonably well with the prediction from the solid surface. The agreement gives some confidence that the quadrupole correction is actually capturing some of the relevant physics for the S-A case.

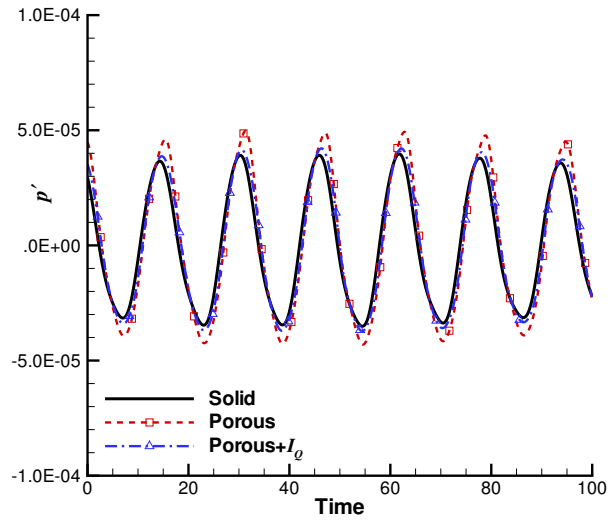

(a) $45^{\circ}$

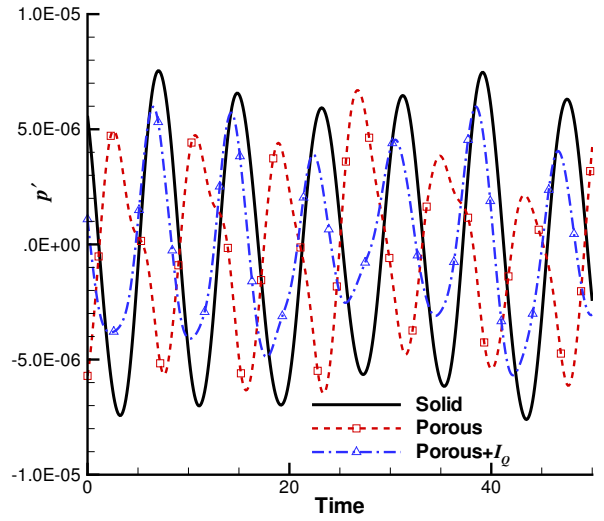

(b) $180^{\circ}$

Figure 16. Time histories of the pressure computed from an S-A calculation of flow over a 3-D cylinder at $M=0.25$. The observers are located on a radius of 100 , and $\theta$ is measured from the upstream direction.

\section{Conclusions}

Using a frozen convection assumption, an FW-H, permeable surface correction has been developed to account for hydrodynamic sources propagating through the surface. The analysis shows that the correction is in the form of an expansion in the Mach number. Test cases have demonstrated that several terms are needed, even at modest Mach numbers. For Mach numbers above 0.5, the expansion is divergent for observers in the upstream direction.

A test case involving vortex shedding revealed that the error caused by the wake passing through the FW-H surface 
was small in comparison to the true noise signal, in agreement with Wang et al. ${ }^{13}$ The error appears to be of the same magnitude as other quadrupole effects, such as propagation through a nonuniform flow. However, the error appears to be nonnegligible in some classes of airframe noise problems, ${ }^{9}$ and further research is needed. Clearly, the error would be important for jet noise problems, but the low Mach number restriction on the current method would need to be overcome to make it viable for most jet problems.

Three-dimensional test cases have emphasized the need for accurate input data. Discrepancies were observed between calculations using detached-eddy simulation and the Spalart-Allmaras RANS turbulence model that resulted in considerable differences in the three-dimensional noise predictions. Further research is needed to determine the relative importance of the different parameters in CFD calculations on noise predictions.

\section{References}

${ }^{1}$ Ffowcs Williams, J. E. and Hawkings, D. L., "Sound Generated by Turbulence and Surfaces in Arbitrary Motion," Philosophical Transactions of the Royal Society, Vol. A264, No. 1151, 1969, pp. 321-342.

${ }^{2}$ Lighthill, M. J., "On Sound Generated Aerodynamically, I: General Theory," Proceedings of the Royal Society, Vol. A221, 1952, pp. 564-587.

${ }^{3}$ Curle, N., "The Influence of Solid Boundaries upon Aerodynamic Sound," Proceedings of the Royal Society, Vol. 231, 1955, pp. 505-514.

${ }^{4}$ Singer, B. A., Lockard, D. P., and Brentner, K. S., "Computational Aeroacoustic Analysis of Slat Trailing-Edge Flow," AIAA Journal, Vol. 38, No. 9, 2000, pp. 1558-1564.

${ }^{5}$ Khorrami, M. R., Singer, B. A., and Berkman, M. E., "Time-accurate Simulations and Acoustic Analysis of Slat Free Shear Layer," AIAA Journal, Vol. 40, No. 7, 2002, pp. 1284-1291.

${ }^{6}$ Casper, J., Lockard, D. P., Khorrami, M. R., and Streett, C. L., "Investigation of Volumetric Sources in Airframe Noise Simulations," AIAA2004-2805, Presented at the AIAA/CEAS Aeroacoustics Conference, Manchester, UK, May 10-12, 2004.

${ }^{7}$ Souliez, F. J., Long, L. N., Morris, P. J., and Sharma, A., "Landing Gear Aerodynamic Noise Prediction Using Unstructured Grids," International Journal of Aeroacoustics, Vol. 1, No. 2, 2002, pp. 115-135.

${ }^{8}$ Lockard, D. P., Khorrami, M. R., and Li, F., "Aeroacoustic Analysis of a Simplified Landing Gear," AIAA-2003-3111, Presented at the 9th AIAA/CEAS Aeroacoustics Conference and Exhibit in Hilton Head, SC, 2003.

${ }^{9}$ Lockard, D. P., Khorrami, M. R., and Li, F., "High Resolution Calculation of a Simplified Landing Gear," AIAA-2004-2887, Presented at the 10th AIAA/CEAS Aeroacoustics Conference, 2004.

${ }^{10}$ Singer, B. A., Lockard, D. P., and Lilley, G. M., "Hybrid Acoustic Predictions," Computers and Mathematics with Applications, Vol. 46, 2003, pp. 647-669.

${ }^{11}$ Mankbadi, R. R., Hayder, M. H., and Povinelli, L. A., "Structure of Supersonic Jet Flow and Its Radiated Sound," AIAA Journal, Vol. 32, No. 5, 1994, pp. 897-906.

${ }^{12}$ Shur, M. L., Spalart, P. R., Strelets, M., and Travin, A. K., "Towards the Prediction of Noise from Jet Engines," International Journal of Heat and Fluid Flow, Vol. 24, 2003, pp. 551-561.

${ }^{13}$ Wang, M., Lele, S. K., and Moin, P., "Computation of Quadrupole Noise Using Acoustic Analogy," AIAA Journal, Vol. 34, No. 11, 1996, pp. 2247-2254.

${ }^{14}$ Crighton, D. G., Dowling, A. P., Ffowcs Williams, J. E., Heckl, M., and Leppington, F. G., Modern Methods in Analytical Acoustics, chap. 11, Springer-Verlag, London, 1992, pp. 334-342.

${ }^{15}$ Farassat, F., "Linear Acoustic Formulas for Calculation of Rotating Blade Noise," AIAA Journal, Vol. 19, No. 9, 1981, pp. $1122-1120$.

${ }^{16}$ Lockard, D. P., "An Efficient, Two-Dimensional Implementation of the Ffowcs Williams and Hawkings Equation," Journal of Sound and Vibration, Vol. 229, No. 4, 2000, pp. 897-911.

${ }^{17}$ Lockard, D. P., "A Comparison of Ffowcs Williams-Hawkings Solvers for Airframe Noise Applications," AIAA Paper 2002-2580, 8th AIAA/CEAS Aeroacoustics Conference, Breckenridge, CO, June 17-19, 2002.

${ }^{18}$ Dowling, A. P. and Ffowcs Williams, J. E., Sound and Sources of Sound, chap. 9, Horwood Publishing, Westergate, 1983, pp. $207-208$.

${ }^{19}$ Gloerfelt, X., Bailly, C., and Juve, D., "Direct Computation of the Noise Radiated by a Subsonic Cavity Flow and Application of Integral Methods," Journal of Sound and Vibration, Vol. 266, 2003, pp. 119-946.

${ }^{20}$ Vatsa, V. N. and Turkel, E., "Assessment of Local Preconditioners for Steady State and Time Dependent Flows," AIAA-2004-2134, Presented at the 34th AIAA Fluid Dynamics Conference and Exhibit, Portland, Oregon, June 28-31, 2004.

${ }^{21}$ Erlebacher, G., Hussaini, M. Y., and Shu, C.-W., "Interaction of a Shock with a Longitudinal Vortex," Journal of Fluid Mechanics, Vol. 337, 1997, pp. 129-153.

${ }^{22}$ Rumsey, C. L., Biedron, R. T., and Thomas, J. L., “CFL3D: Its History and Some Recent Applications,” TM 112861, NASA, May 1997, presented at the Godonov's Method for Gas Dynamics Symposium, Ann Arbor, MI.

${ }^{23}$ Krist, S. L., Biedron, R. T., and Rumsey, C. L., "NASA Langley Research Center: Computational Aerosciences Branch," CFL3D User's Manual (Version 5), 1997.

${ }^{24}$ Menter, F. R., “Zonal Two-equation k-omega Turbulence Models for Aerodynamic Flows,” AIAA-1993-2906, 1993. 1605.

${ }^{25}$ Menter, F. R., "Two-equation Eddy-viscosity Turbulence Models for Engineering Applications,” AIAA Journal, Vol. 32, No. 8, 1994, pp. 1598-

${ }^{26}$ Spalart, P. R. and Allmaras, S., “A One-Equation Turbulence Model for Aerodynamic Flows,” La Recherche Aerospatiale, Vol. 1, No. 1, 1994, pp. 5-21.

${ }^{27}$ Spalart, P. R., "Strategies for Turbulence Modeling and Simulations," International Journal of Heat and Fluid Flow, Vol. 21, 2000, pp. 252-263.

${ }^{28}$ Squires, K. D., Forsythe, J. R., Morton, S. A., Strang, W. Z., Wurtzler, K. E., Tomaro, R. F., Grismer, M. J., and Spalart, P. R., "Progress on Detached-Eddy Simulation of Massively Separated Flows," AIAA-02-1021, Presented at the 40th AIAA Aerospace Sciences Meeting and Exhibit in Reno, NV, 2002. 